\title{
Manufacturing of Medical Implants by Combination of Selective Laser Melting and Laser Ablation
}

\author{
S. Hallmann ${ }^{1} \cdot$ P. Glockner ${ }^{1}$ - C. Daniel ${ }^{1}$. \\ V. Seyda ${ }^{1} \cdot$ C. Emmelmann ${ }^{1}$
}

\begin{abstract}
The perfect fit of hip stem prostheses is supposed to have positive effects on their lifetime performance. Moreover, the ingrowth of tissue into the surface of the implant has to be assured to create a firm and load bearing contact. For the manufacturing of customized hip stem prostheses, the technology of Selective Laser Melting has shown promising results. Poor surface quality, however, makes it necessary to finish up the part by e.g., sand blasting or polishing. With the use of laser ablation for postprocessing, reproducible and functionalized surface morphologies might be achievable. Hence, with the motive to produce customized hip stem prostheses, a combined process chain for both mentioned laser technologies is developed. It is examined what type of surface should be produced at which part of the process chain. The produced implants should contain the demanded final surface characteristics without any conventional post-processing. Slight advantages for the Selective Laser Melting regarding the accuracy for different geometrical structures of $400 \mu \mathrm{m}$ depth were observed. However, an overall improvement of surface quality after the laser ablation process in terms of osseointegration could be achieved. A complete laser based production of customized hip stem implants is found to be with good prospects.
\end{abstract}

Keywords Selective Laser Melting $\cdot$ Laser ablation $\cdot$ TiAl6V4 $\cdot$ Hip stem prosthesis

\section{Introduction}

With Selective Laser Melting (SLM) creation of free-formed structures with complex geometry is possible. Especially, customized medical implants with lot size one and high geometrical complexity are very interesting for the application of this

\section{S. Hallmann \\ s.hallmann@tuhh.de}

1 Institute of Laser and System Technologies (iLAS), Hamburg University of Technology (TUHH), Denickestr. 17, 21073 Hamburg, Germany 
manufacturing technology [1]. The SLM process includes the successive melting of single-component metal powder layer by layer [2]. The energy of the laser beam fully melts the powder, which solidifies and fuses with the previously molten layer. SLM is suitable for the manufacturing of functional components, has an optimal material consumption and opens up new possibilities for the design of products. However, the surface morphology of implants produced with SLM does not meet medical requirements in terms of reproducibility and specific surface roughness [3]. The so called staircase-effect due to a rather large layer thickness limits the achievable surface quality. Concerning the geometry a probable distortion caused by internal stresses has to be avoided [4]. In addition, loose powder particles may stick on the surface, so that a manual cleaning is necessary [3].

It is important to consider that osseointegration particularly depends on the initial surface of an implant [5]. In order to achieve desirable implant surfaces, different approaches with diverse geometrical surface structures of optimized size are used [6]. Therefore, the surfaces of conventionally forged or milled implants have to be extensively post-processed in order to meet the requirements like a specific surface roughness. Conventional methods like sand blasting or polishing are neither creating reproducible geometrical patterns nor are they applicable to only specific areas of an implant. The use of pulsed lasers for the precise ablation of patterns with high resolution seems to be a promising alternative. Applications for pulsed laser systems are found e.g., in fundamental research and material processing as well as medical procedures [7]. Due to their low acquisition cost and marginal effort for maintenance nanosecond lasers have an economic benefit compared to lasers with shorter pulse duration [8]. During the laser ablation process material is removed from a surface using high-intensity laser pulses. In particular by ablating with nanosecond pulses the process is dominated by heat conduction, melting, evaporation and plasma formation [9]. The irradiated energy of the ns-pulse is absorbed by the solid and the evolving thermal wave propagates into the material [10]. Depending on the achieved temperature the material is molten up or a vapor plume is ejected above the irradiated zone which can become ionized producing plasma. The vapor can further absorb and disperse the laser beam, changing the actual flux received by the surface [11]. Vapor and plasma recoil pressure lead to a partial removal of material either in vapor or liquid phase whereas some melt remains at the surface due to tension forces [9, 12]. The layer-by-layer removal of material with laser ablation is also suitable for very hard materials. Limitations can be seen in a relatively long processing time as well as in heat affected zones and e.g., inaccuracies when creating vertical walls [13].

In this context, the present investigation exposes the potential of laser material processing in the field of medical implant production. Specific design characteristics will be implemented on test specimens derived from today used shafts of hip joint prostheses. It is elaborated where the information and the material path of both mentioned technologies respectively could be connected in the best way. Possible synergy effects and a reduction of drawbacks of SLM and laser ablation are expected from this combination. Therefore, the specimens will be manufactured by SLM with deduced surface structures on one side. On the other side of the identical specimens the structures are laser ablated. Afterwards the achieved results are compared with each other. 


\section{Material \& Methods}

The data preparation for the SLM process includes different computer aided design (CAD) software. The same created initial data are also used for the laser ablation process. After manufacturing the parts by SLM, the laser ablation process follows. Figure 1 shows the combined process chain of both processes. Producing specimens with or without surface structure will not significantly affect the processing time of the SLM process. The time needed for the laser ablation is just added to the total processing time. In addition, in one SLM process-run several parts can be built simultaneously while in laser ablation parts have to be processed sequentially.

The powder material which was used for this study is the alloy TiAl6V4 with a mean particle size of $49 \mu \mathrm{m}$ [14]. The SLM process was carried out on a commercially available machine with a $200 \mathrm{~W}$ continuous wave (CW) Ytterbium (Yb) fiber laser and a focal spot diameter of $100 \mu \mathrm{m}$. Laser parameters set to a scan speed of $1,250 \mathrm{~mm} / \mathrm{s}$, laser power of $170 \mathrm{~W}$ and hatch distance of $100 \mu \mathrm{m}$ lead to a layer thickness of $30 \mu \mathrm{m}$. For laser ablation a ns pulsed $\mathrm{Yb}$ fiber laser with $200 \mathrm{~W}$ average output power and focal spot of approximately $60 \mu \mathrm{m}$ was used. For a precise positioning of the specimens the machining setup consists of five $\mathrm{CNC}$ axes, three linear and two rotational. The scan head itself provides three additional optical axes. A focal length of $163 \mathrm{~mm}$ results in a maximum scan field size of $60 \mathrm{~mm} \times 60 \mathrm{~mm}$ in the $\mathrm{x}$ y-plane. Laser ablation was conducted with a pulse duration of $120 \mathrm{~ns}$ and pulse energy of $0.28 \mathrm{~mJ}$. Furthermore, a scan speed of $3,000 \mathrm{~mm} / \mathrm{s}$ and a track as well as pulse distance of $25 \mu \mathrm{m}$ were set. These parameters resulted in an ablation per layer with a thickness of $1 \mu \mathrm{m}$.

Specimens were designed and built in the SLM process with three different directions to the base plate, horizontal $\left(0^{\circ}\right)$, sloped $\left(45^{\circ}\right)$ and vertical $\left(90^{\circ}\right)$ due to expected influences on each surface quality. The geometrical surface structures of the specimens were deduced from the mostly implanted hip joint prostheses shafts according to the Swedish hip arthroplasty register 2011 [15].

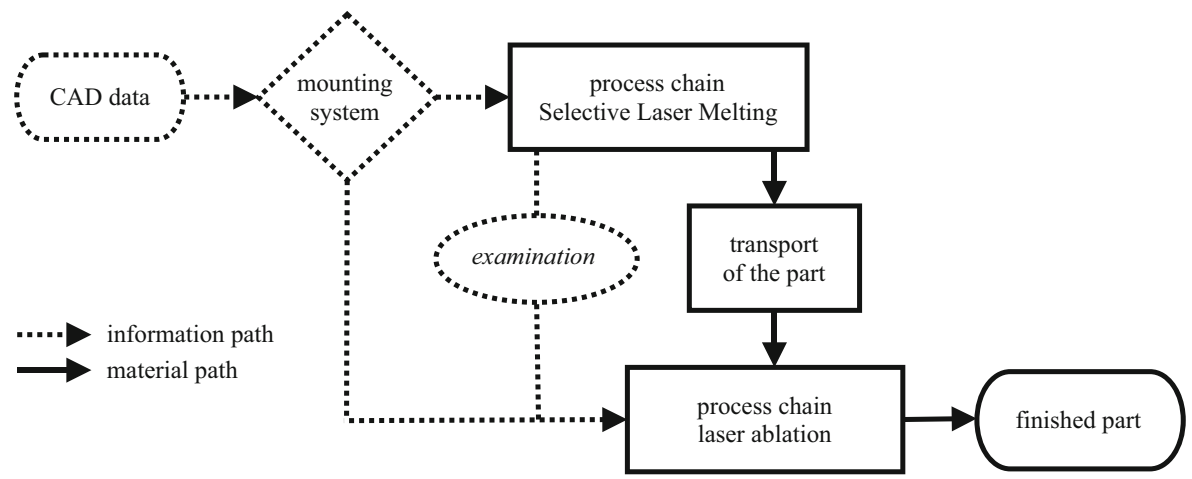

Fig. 1 The combined process chain of SLM and laser ablation is shown. The type of part fixation has to be chosen first in order to fit for both processes. Afterwards the SLM process can be started and the manufactured part is transported to the second machine. There the laser ablation process is performed 

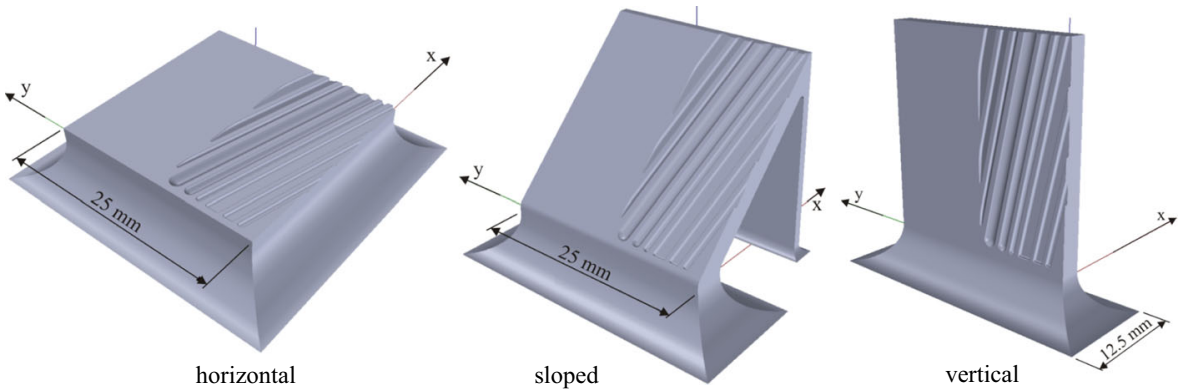

Fig. 2 The different designs of the specimens are displayed. On the right-hand side of each specimen the surface structures were directly produced by SLM. The left-hand side of each specimen is characterized by a flat molten surface in order to ablate the identical surface structures

They contain triangles, rectangles and a rounded geometry. These structures are $400 \mu \mathrm{m}$ deep and have a width of $600 \mu \mathrm{m}, 1,100 \mu \mathrm{m}$ and 1,600 $\mu \mathrm{m}$. On the right-hand side of each specimen (Fig. 2) the surface structures were directly produced by SLM. The left-hand side of each specimen is characterized by a flat molten surface in order to ablate the same surface structures. The positioning of the specimens during the laser ablation process were set up in such a way that the laser beam radiates with an incident angle of $90^{\circ}$ to the initial surface.

For surface characterization of the laser molten and ablated specimens 3D images were created by a confocal laser scanning microscope and additional 2D images were made by an optical microscope. The surface roughness was measured according to ISO 25178 [16]. To exclude statistical variation the mean values $\mathrm{S}_{a}$ and $\mathrm{S}_{z}$ were measured at 18 different positions.

The different specimens were named with the following key for technology $(\mathrm{m}$ : molten or a: ablated)-direction ( $h$ : horizontal, $s$ : sloped or $v$ : vertical)-geometry ( $t$ : triangular, re: rectangular or ro: round). For instance, $\mathrm{m}$-h-t refers to the horizontally laser molten specimen with triangular structure.

For the assessment of the surface structures, mean profiles were measured out of 19 cross sections on a length of $2.5 \mathrm{~mm}$ each. The profiles were processed further by exporting their height information. This processing was necessary to get results similar

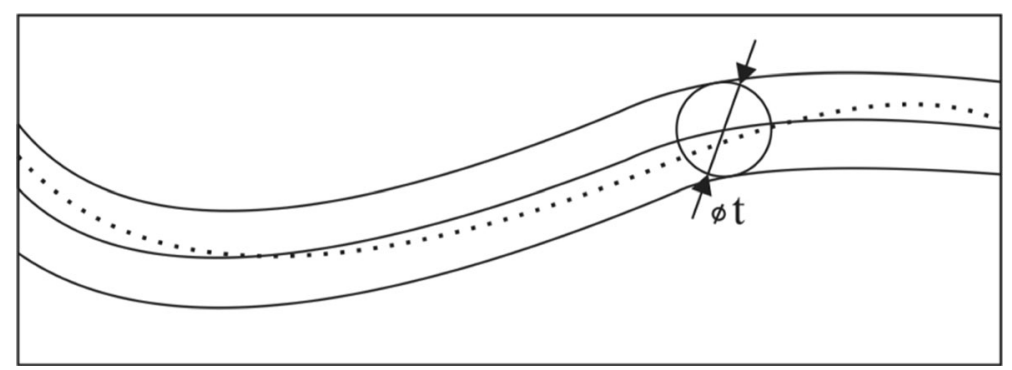

Fig. 3 The tolerance of a part can be defined, as explained in ISO 1101 [17], by moving a circle along the target profile. During this investigation, the building inaccuracy was assessed as the area which included all measured profiles of one geometry type 
to those explained in ISO 1101 [17]. As sketched in Fig. 3 the area of tolerance is defined by the outer contour of a circle moved over the target profile. If a measured profile lies inside this defined range, its geometry can be tolerated. The maximum inaccuracy of the produced structures was detected and compared similar as the tolerance in ISO 1101. The area of inaccuracy surrounding the target CAD profile included all assessed profiles of a specific surface geometry.

\section{Results}

The results of the surface roughness measurements examined in the widest rectangular cavity are displayed in Figs. 4 and 5 respectively. The lowest value of $\mathrm{S}_{a}=2.72 \pm 0.08 \mu \mathrm{m}$ was measured on $\mathrm{m}-\mathrm{h}$. The surface of $\mathrm{m}-\mathrm{v}$ showed a roughness of $\mathrm{S}_{a}=15.32 \pm 1.05 \mu \mathrm{m}$. The difference of $\mathrm{S}_{a}$ values for the SLM specimens were up to $13.65 \mu \mathrm{m}$. The laser ablated surfaces featured $\mathrm{S}_{a}$ values of maximum $8.1 \pm 1.48 \mu \mathrm{m}$ for a-s and of minimum $5.37 \pm 0.73 \mu \mathrm{m}$ for a-h. This results in a range of $\mathrm{S}_{a}$ of $4.94 \mu \mathrm{m}$ comparing the different building directions.

The highest $\mathrm{S}_{z}$ value for the SLM processed surfaces was measured on $\mathrm{m}$-s with $173.04 \pm 11.65 \mu \mathrm{m}$. In contrast, the lowest $\mathrm{S}_{z}=64.27 \pm 6.64 \mu \mathrm{m}$ was measured on $\mathrm{m}-\mathrm{h}$. Thus, the $S_{z}$ values for the SLM surfaces deviated by $113.78 \mu \mathrm{m}$ in total. The lowest value for the laser ablated surfaces was observed for a-h with $\mathrm{S}_{z}=71.65 \pm 7.97 \mu \mathrm{m}$ and the highest $S_{z}$ value of $136.32 \pm 12.36 \mu \mathrm{m}$ for a-s. Total deviation for laser ablation on specimens of different building direction is $69.06 \mu \mathrm{m}$.

The morphology of the SLM and the laser ablated surfaces is shown in Figs. 6 and 7 respectively. The melt pattern of the CW-laser is clearly visible on the images of $\mathrm{m}-\mathrm{h}$ in Fig. 6a. Small amounts of partially molten powder particles remain on the surface. In Fig. $6 \mathrm{~b}$ the surface of $\mathrm{m}-\mathrm{v}$ is shown. The peaks seen in the $3 \mathrm{D}$ image confirm a high $\mathrm{S}_{a}$ value of $15.32 \pm 1.05 \mu \mathrm{m}$.

The surface of a-h shows no longer melt pattern (Fig. 7a). Moreover the ablated surface is rougher than the initial one. In the image of the optical microscope craters in the size of several tenths of microns are visible. In contrast, a smoother surface can be observed in the image of a-v (Fig. 7b). The peaks, formerly visible after the SLM process (Fig. 6b), are nearly

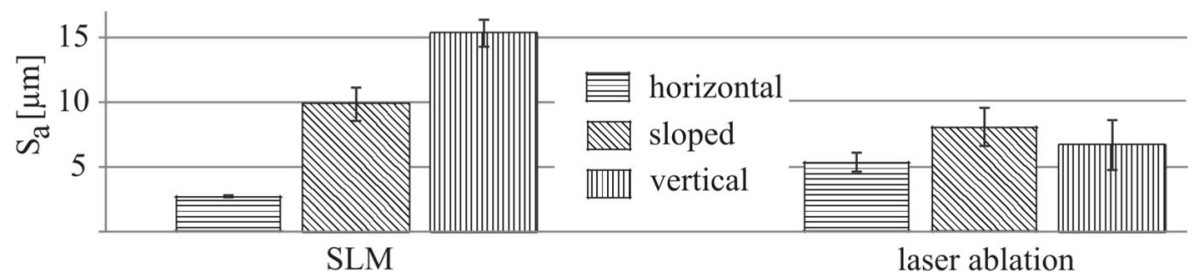

Fig. 4 The resulting $\mathrm{S}_{a}$ values of the surface roughness measurements according to ISO 25178 are shown. The $\mathrm{S}_{a}$ values of the SLM surfaces contained the overall maximum and minimum. The surfaces after laser ablation showed a much more equalized result. Furthermore, a lower dependency to the building direction is visible in contrast to the SLM surfaces 


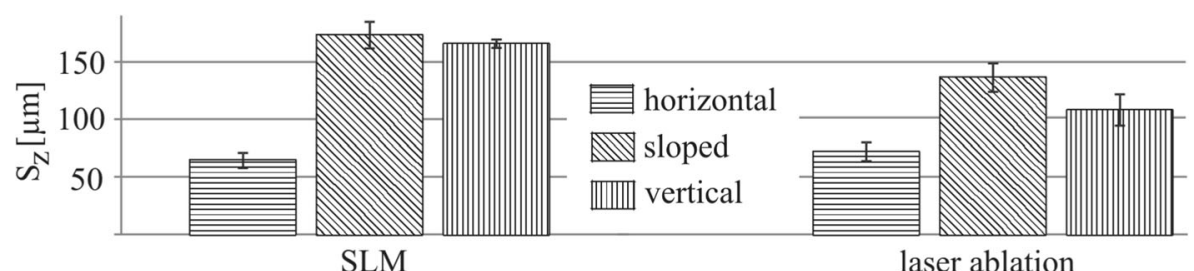

Fig. 5 The $S_{z}$ values for the SLM surfaces have a range of $113.7 \mu \mathrm{m}$. The $S_{z}$ values after the laser ablation process increased for the horizontal specimen but decreased for the sloped and the vertical surfaces

removed. The ablated surfaces further show similar roughness patterns independent of the building direction.

In Fig. 8 deviation of the flank angle on $m-h-t$ is visible. The slope of the triangle was affected by the stair-case-effect. The mean profile over a length of $4.5 \mathrm{~mm}$ (profile 6) confirms this effect. The resulting inaccuracy of this structure amounts to $75 \mu \mathrm{m}$ which is drawn as light grey area around the target profile. Another deviation of flank angle can be observed in Fig. 9, where the edge of $\mathrm{m}-\mathrm{v}-\mathrm{re}$ is shown. Moreover, the measurements are variable (profiles 1-5). The rectangular structure has an approximated inaccuracy of $130 \mu \mathrm{m}$.

In Fig. 10 the profile of a-h-t is shown. A shifting of the ground to the right is visible (Fig. 10a). There is hardly variation of the measured profiles over the length of the specimen. The result is also visible at the lateral cut (Fig. 10b). Another characteristic is the existence of elevated edges, which can be observed at the border of the ablated structure. Figure 11 of a-v-re shows that the lower edge is deeper ablated than the rest of the ground. This behaviour is also significant for all rectangular structures.

A comparison of the results concerning the inaccuracy is shown in Fig. 12. This figure shows the maximum deviation of the manufactured surface structures, independent from the width. The results of different geometrical structures produced within one technology are differing widely. The highest deviation from the target profile was measured at a-h-t and a-s-t with $155 \mu \mathrm{m}$ and
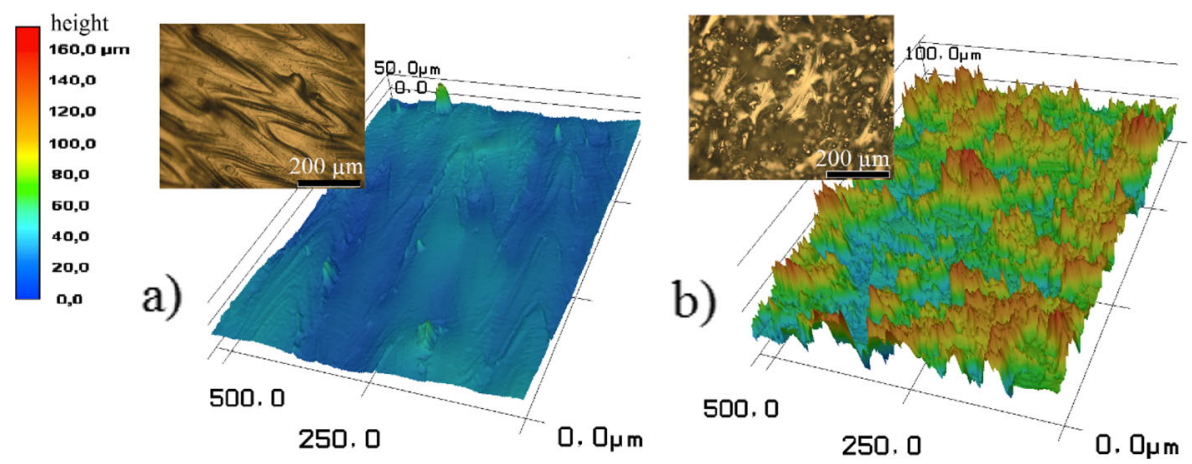

Fig. 6 a) The 3D image of $m-h$ is shown supported by a $2 D$ image. b) The surface of $m-v$ is displayed 

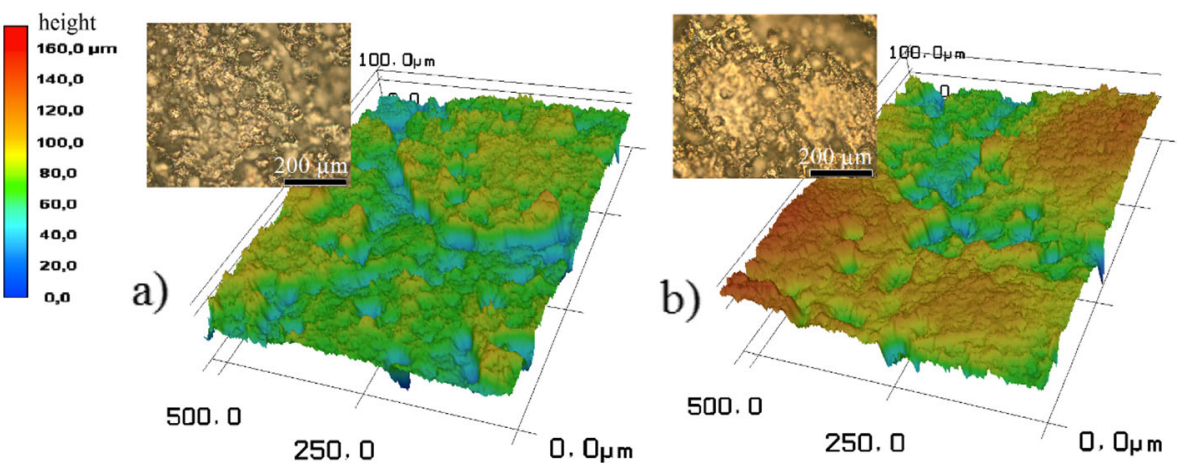

Fig. 7 a) Images of $\mathrm{m}-\mathrm{h}$, which was afterwards laser ablated (a-h). b) The images of a-v show a similar morphology although the initial surfaces featured a different roughness

$160 \mu \mathrm{m}$ respectively. The structure at m-s-ro showed the lowest deviation with $60 \mu \mathrm{m}$.

\section{Discussion}

The surface roughness of SLM specimens strongly depends on the building direction. The peaks visible in Fig. $6 b$ result in high $S_{a}$ and $S_{z}$ values for $m-v$. Powder particles which were fused to the solid part during the processing are identified. Comparing the different SLM specimens (Fig. 6a, b), different surface roughness and therefore different optical characteristics are present. As the laser ablation process equalizes the molten surfaces, an overall better predictable surface roughness can be achieved. A strong bone response with moderately rough surfaces $\left(\mathrm{S}_{\mathrm{a}}=1-2 \mu \mathrm{m}\right)$ was demonstrated by a large number of investigations [18]. In order to reduce the surface roughness through laser ablation, a solution may be found in changing the laser parameters. However, the surface roughness of a cementless hip stem prosthesis which is already in medical use and which was analysed in this examination, showed values of $\mathrm{S}_{\mathrm{a}}$ $=8.54 \pm 1.21 \mu \mathrm{m}$ and $\mathrm{S}_{\mathrm{z}}=98.87 \pm 15.81 \mu \mathrm{m}$. This indicates that laser ablation may be well suited as a tool for surface finishing for currently used hip joint prostheses.
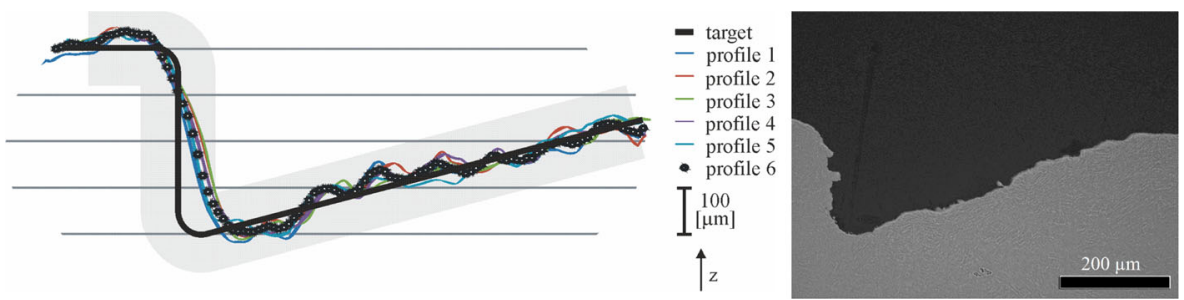

Fig. 8 The result for $\mathrm{m}$-h-t is displayed. The deviation of the molten structure is visible at the lowest point of the target profile. Furthermore the stair-case-effect is apparent which led to a poor representation of the slope 


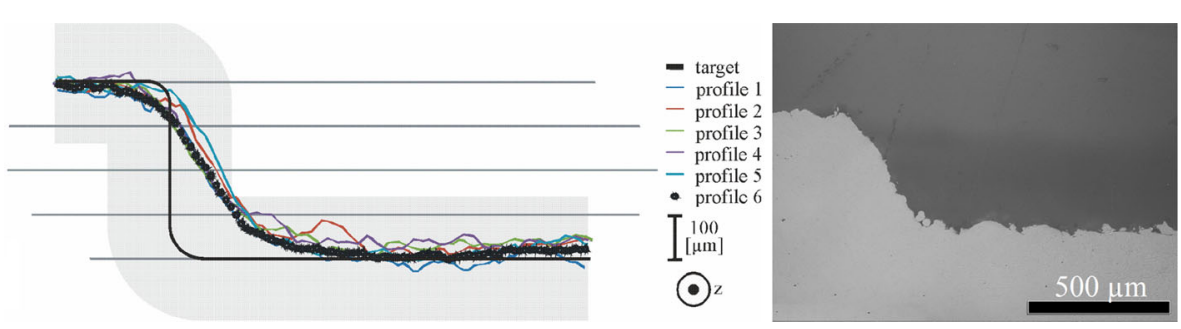

Fig. 9 The result for $m-v-r e$ is shown. The deviation of the flank angle is around $30^{\circ}$. The measured profiles show less homogeneity

The resolution of the SLM geometrical structures depends both on the beam size [4] and the layer thickness. The structure in Fig. 8 is a good example for the stair-caseeffect. The discrete layer thickness of $30 \mu \mathrm{m}$ resulted in a stepwise approximation of the slope. Also poor representation of m-v-re is observed (Fig. 9). This result might be improved by a scanner control with higher precision.

The shifting of a-h-t (Fig. 10) can be caused by ablating vertical walls with a Gaussian laser beam profile. The laser ablates a cavity in the size of the cross section of its intensity profile. The following laser pulse in the next layer is projected on a bigger area with the effect of lowering the energy density. This influences the ablation process resulting in the tilted triangle. In addition, the cavity is affected by melt deposit. The elevated edges, the so called burr formation, are present at all ablated cavities and evolve from accumulation of thin molten layers. The melt was accelerated by vapour pressure and stuck on the sidewalls of the cavities [19]. Removing or even avoiding molten material during the ablation process will result in a higher quality by decreasing the achieved deviation. By comparing the profiles 1-6 of Fig. 10a, it can be seen that a stable ablation within a range of 15 to $30 \mu \mathrm{m}$ was achieved. The deviation of the flank angle at the ablated rectangular structure (Fig. 11) of all specimens is also affected by the laser beam profile. Furthermore, the partially reflected laser beam is guided towards the bottom of the cavity and caused ablation at the edge of the ground.

Combining SLM and laser ablation results in a building inaccuracy which is nearly similar for both technologies (Fig. 12). However, slightly better results for the SLM structures were found. The highest deviation of $130 \mu \mathrm{m}$ was obtained on $\mathrm{m}-\mathrm{v}-\mathrm{re}$ and $\mathrm{m}-\mathrm{s}-\mathrm{re}$. This was a consequence of a limited precision

a)

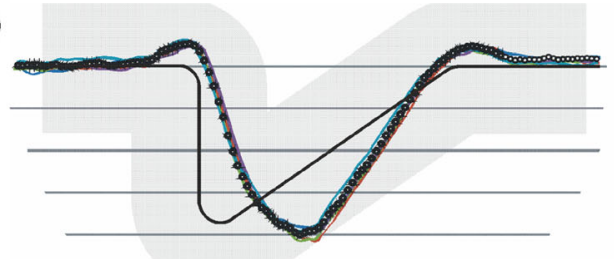

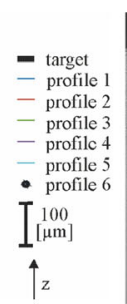

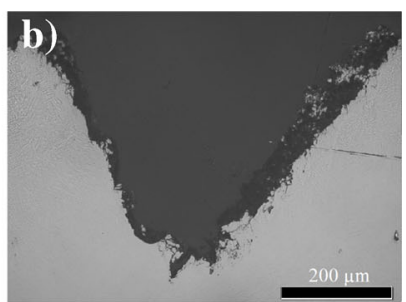

Fig. 10 a) The surface of a-h-t shows a shifting of the ground to the right. This is due to the deviation of the wall on the left side. b) The effect is as well displayed at the image of the lateral cut through 

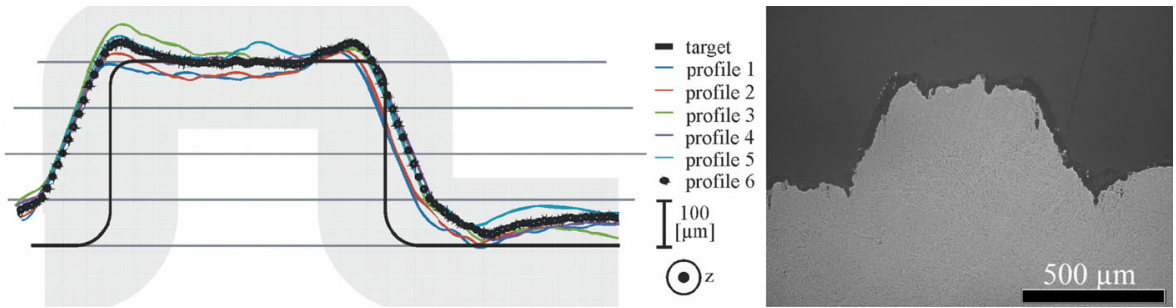

Fig. 11 The surface of a-v-re is shown. Elevated edges at the top of the structure are visible as well as higher ablation depth at the lower edge

of the scanner control. A high surface roughness increases deviation as well. The highest deviation of laser ablation was found on a-h-t and a-s-t. It occurred due to reflection mechanisms and burr formation. However, the molten material might be easily removed after ablation. When using laser ablation for surface finishing melt deposits are not desired. They can be avoided by reducing the energy density slightly above the ablation threshold [20]. The drawback will be a significant reduction of the ablation rate. By choosing ultrashort pulses in the range of pico- or femtoseconds the penetration depth decreases. This would lead to a reduced interaction volume between material and laser radiation. Thus a more precise result could be achieved [19]. Shortening pulse duration means also an increased ratio of evaporated material and therefore decreased amount of melt. However, this will result in a higher technical effort regarding the laser source and will therefore lead to a significant increase in costs. Thus the pulse duration should always be chosen as short as necessary in order to achieve a satisfying result [20] but as long as possible to keep costs at a low level. For the manufacturing of hip joint prosthesis shafts an accuracy e.g., in a micrometre range would not lead to better long-term performance. For implants the use of ns pulsed lasers is regarded as suitable. However, vertical walls in the hip stem prosthesis are required to transfer the mechanical load normally rather than by shear stresses at the surface of the implant. So the correct realization of this geometry becomes necessary.

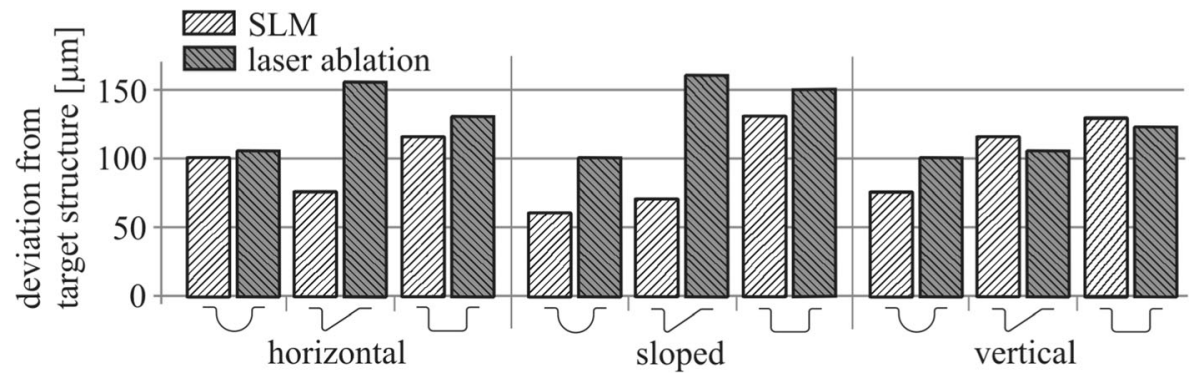

Fig. 12 The overview of the maximum values of the manufacturing inaccuracy is shown, independent from the width of the cavity. Differences depending on the building direction, target structure and manufacturing technology occurred 


\section{Conclusion}

The process chain developed during this investigation, which includes the combination of SLM and laser ablation, was performed successfully. The surface roughness of the SLM specimens showed differences regarding the building direction. After a following laser ablation process, however, those differences were reduced significantly. With a treatment by laser ablation the molten surface is improved towards the roughness already used for implant material. With regards to the differing processing times and the higher accuracy of the molten geometrical structures, it is suggested to build individual hip stem prostheses including the structures with SLM. This applies for geometrical structures of the dimensions which were assessed in this investigation. A laser ablation process after SLM can then be performed to create defined surface roughness and to finally adjust the roughness and dimensions of the additively manufactured structures.

\section{References}

1. Kruth, J.P., Badrossamay, M., Yasa, E. Deckers, J., Thijs, L., Van Humbeek, J.: Part and material properties in selective laser melting of metals, 16th International Symposium on Electromachining (2010)

2. Gebhardt, A.: Generative Fertigungsverfahren: Rapid Prototyping - Rapid Tooling - Rapid Manufacturing, Carl Hanser Verlag, München, 499 p. (2007)

3. Abele, E. and Reihart, G.: Zukunft der Produktion, Carl Hanser Verlag, Munich, 244 p. (2011)

4. Meiners, W.: Direktes Selektives Laser Sintern einkomponentiger metallischer Werkstoffe, Dissertation at the RWTH Aachen, 126 p. (1999)

5. Khanuja, H.S., Vakil, J.J., Goddard, M.S., Mont, M.A.: Cementless femoral fixation in total Hip arthroplasty. J Bone Joint Surg 93, 500-509 (2011)

6. Harrysson, O. L. A., Cormier, D. R.: Direct Fabrication of Custom Orthopedic Implants using Electron Beam Melting Technology, Chapter 9, Advanced Manufacturing Technology for Medical Applications, John Wiley \& Sons, Ltd., 193-208 (2006)

7. Meijer, J., Du, K., Gillner, A., Hoffmann, D., Kovalenko, V.S., Masuzawa, T., Ostendorf, A., Poprawe, R., Schulz, W.: Laser machining by short and ultrashort pulses, state of the art and new opportunities in the age of the photons. CIRP Annals-Manuf Technol 51(2), 531-550 (2002)

8. Fu, Z., Wu, B., Gao, Y., Zhou, Y., Yu, C.: Experimental study of infrared nanosecond laser ablation of silicon: The multi-pulse enhancement effect. Appl Surf Sci 256(7), 2092-2096 (2010)

9. Leitz, K.H., Redlingshöfer, B., Reg, Y., Otto, A., Schmidt, M.: Metal ablation with short and ultrashort laser pulses. Phys Procedia 12, 230-238 (2011)

10. Chichkov, B.N., Momma, C., Nolte, S., Von Alvensleben, F., Tünnermann, A.: Femtosecond, picosecond and nanosecond laser ablation of solids. Applied Physics A 63(2), 109-115 (1996)

11. Brown, M. S. and Arnold, C. B.: Fundamentals of laser-material interaction and application to multiscale surface modification, In Laser Precision Microfabrication, Springer, pp. 91-120 (2010)

12. Schäffer, R. D.: Fundamentals of Laser Micromachining, CRC Press - Taylor \& Francis Group, Boca Raton, 238 p. (2012)

13. Yasa, P., Kruth, J.-P.: Investigation of laser and process parameters for selective laser erosion. Precis Eng 34, 101-112 (2010)

14. Seyda, V., Herzog, D., Emmelmann, C., Jahn, S., Sändig, S.: On the treatment of Ti6-Al-4V Powder in Laser Melting, DDMC Conference 2014, Berlin (2014)

15. Garellik, G., Karrholm, J., Rogmark, C., Rolfson, O., Herberts, P.: Swedisch Hip Arthroscopy Register, Annual Report 2011, 136 p. (2011)

16. ISO 25178-3: Geometrical product specifications (GPS) - Surface texture: Areal - Part 3: Specification, https://www.iso.org, 18 p. (2012)

17. ISO 1101: Geometrical product specifications (GPS) - Geometrical tolerancing - Tolerances of form, orientation, location and run-out, https:/www.iso.org, 103 p. (2012) 
18. Dohan Ehrenfest, D.M., Coelho, P.G., Kang, B.-S., Sul, Y.-T., Albrektsson, T.: Classification of osseointegrated implant surfaces: materials, chemistry and topography. Trensds in Biotechnol 28(4), 198-206 (2010)

19. Vandenbroucke, B., Kruth, J.-P.: Selective laser melting of biocompatible metals for rapid manufacturing of medical parts. Rapid Prototyp J 13(4), 196-203 (2007)

20. Dausinger, F., Hügel, H., Konov, V.: Micro-machining with ultrashort laser pulses: From basic understanding to technical applications, ALT'02 International Conference on Advanced Laser Technologies, SPIE Proceedings, Vol. 5147, doi: 10.1117/12.537496 (2003) 